nephron

Practice
Nephron 2019;143:211-216

DOI: $10.1159 / 000501207$
Received: April 12, 2019

Accepted after revision: May 22, 2019

Published online: June 14, 2019

\title{
Differentiating Acute Interstitial Nephritis from Acute Tubular Injury: A Challenge for Clinicians
}

\author{
Dennis G. Moledina ${ }^{a, b} \quad$ Chirag R. Parikh ${ }^{c}$ \\ a Section of Nephrology, Department of Internal Medicine, Yale School of Medicine, New Haven, CT, USA; \\ bProgram of Applied Translational Research, Department of Internal Medicine, Yale School of Medicine, \\ New Haven, CT, USA; ' Division of Nephrology, School of Medicine, Johns Hopkins University, Baltimore, MD, USA
}

\section{Keywords}

Acute renal injury · Fibrosis · Kidney · Nephrology

\begin{abstract}
Background: Differentiating etiologies of acute kidney injury (AKI) is critical in determining the course of care in clinical practice. For example, acute interstitial nephritis (AIN) requires withdrawal of the offending drug and immunosuppressive therapy, while acute tubular injury (ATI) does not have any disease-specific therapies. Failure to distinguish AIN from ATI in a timely manner can lead to kidney fibrosis and chronic kidney disease. In this review, we discuss current tests and novel biomarkers to distinguish ATI from AIN. Summary: In a prospective cohort study of 32 participants with AIN and 41 with ATI, clinical features and current, laboratory tests did not provide sufficient distinction between the 2 subpopulations of AKI. The findings in our cohort are consistent with our review of the literature. Given the limitations of clinical features and laboratory assessments, clinical practice relies on kidney biopsy for histological diagnosis, which is not always feasible, and is associated with bleeding complications in high-risk populations. In addition, histological diagnosis is prone to sampling errors and inter-rater variability. In the interest of identifying a novel biomarker,
\end{abstract}

we compared urine and plasma levels of cytokines in the Th1, Th2, and Th9 pathways, which have been implicated in the pathogenesis of AIN. Urine TNF- $\alpha$ and interleukin-9 were higher in AIN participants than in ATI controls and helped discriminate AIN from ATI (area under curve 0.83 [0.730.92]). Key Messages: Differentiation between AIN and ATI in patients with AKI using currently available tests is challenging. Urine TNF- $a$ and interleukin-9 may help clinicians separate AIN from ATI.

(c) 2019 S. Karger AG, Basel

\section{Introduction}

Acute kidney injury (AKI) consists of a group of diseases characterized by loss of kidney function. A major challenge in the clinical care of patients with AKI is differentiating between its underlying etiologies such as acute tubular

Contribution from the AKI and CRRT 2019 Symposium at the 24th International Conference on Advances in Critical Care Nephrology, Manchester Grand Hyatt, San Diego, CA, USA, February 26 - March 1, 2019. This symposium was supported in part by the NIDDK funded University of Alabama at Birmingham-University of California San Diego O'Brien Center for Acute Kidney Injury Research (P30DK079337).

\section{KARGER}

(c) 2019 S. Karger AG, Basel

E-Mail karger@karger.com

www.karger.com/nef
Chirag R. Parikh, MD, PhD

Ronald Peterson Professor of Medicine, Division of Nephrology Johns Hopkins University School of Medicine

1830 E. Monument Street, 4th Floor, Suite 416, Baltimore, MD 21287 (USA)

E-Mail chirag.parikh@jhmi.edu 
injury (ATI) and acute interstitial nephritis (AIN). Timely differentiation between these etiologies of AKI is critical due to differences in their management. ATI does not have any disease-specific therapies. However, AIN is treated through withdrawal of the offending drug and immunosuppressive therapy. Failure to recognize and treat AIN promptly could lead to fibrosis, permanent kidney damage, and progression to chronic kidney disease (CKD) [1].

In the present report, we review data from published studies evaluating clinical features and diagnostic tests that could help a clinician differentiate between AIN and ATI. In addition, we present data from a prospective, observational study of participants who underwent a kidney biopsy for evaluation of AKI between 2015 and 2018 at 2 Yale University-affiliated hospitals [2]. We discuss clinical features, traditional tests (blood eosinophils, urinalysis, and urine microscopy), histological features, and novel biomarkers for differentiating AIN from ATI.

\section{Clinical Features}

Due to varying clinical features, latent period of disease presentation, and variable degree of kidney dysfunction, AIN is often confused with other kidney diseases such as ATI or progressive CKD. A few decades ago, AIN occurrence was predominantly by medications such as beta-lactam antibiotics and sulfur-containing drugs and the patient presented with acute or sub-acute onset of allergic features such as fever, rash, and eosinophilia within a few days of starting the drug. Recently, however, other medication classes such as proton pump inhibitors (PPI), nonsteroidal anti-inflammatory drugs, and cancer immunotherapy agents have become common causes of AIN. AIN from these drugs do not present with the same allergic features and the clinical presentation is protracted. In fact, the classic triad of fever, rash, and eosinophilia was reported in only $10 \%$ of antibiotic-induced AIN cases, and these features were not present in any of the PPI-induced AIN cases [3]. Furthermore, AIN cases induced by these new medication classes have significantly longer latent periods than cases caused by beta-lactam antibiotics or sulfur-containing drugs. For example, studies demonstrated that AIN occurs 8-15 days after antibiotic initiation, 76 days after nonsteroidal anti-inflammatory drugs initiation, and 234 days after PPI initiation [3,4]. This subacute to chronic clinical onset of renal dysfunction in AIN can be hidden under the natural history of progressive $\mathrm{CKD}$, delaying AIN diagnosis and treatment. In a study of biopsy-proven AIN, only half of the participants exhibited a sharp increase in serum creatinine in $48 \mathrm{~h}$ to 7 days (AKI), whereas over $90 \%$ exhibited a serum creatinine increase over a longer time frame of $<3$ months (Acute Kidney Disease) [5].

\section{Noninvasive Diagnostic Tests}

There is no reliable, noninvasive diagnostic test for clinical diagnosis of AIN. Urine eosinophil testing was once considered a diagnostic test for AIN. However, a recent study showed that the sensitivity and specificity of urine eosinophil testing were 31 and $68 \%$, respectively, indicating that this test could not reliably differentiate AIN from other causes of AKI [6]. Urine eosinophils were found not only in AIN but also in cases with glomerulonephritis, atheroembolic disease, multiple myeloma, and sometimes in cases with ATI. Another clinical test of interest for AIN diagnosis is urine sediment examination for sterile pyuria and white blood cell casts. However, one case series in AIN showed that only $15 \%$ had white blood cell casts [6]. Furthermore, the study did not evaluate the presence of these casts in other causes of AKI, and this method has not been systematically evaluated. While markers of tubular injury and dysfunction such as low-grade proteinuria and elevated urine levels of biomarker neutrophil gelatinase-associated lipocalin are present in AIN, these are not specific for AIN and are also elevated in other causes of AKI most notably in ATI [7]. Gallium-67 scanning has been proposed as a test of AIN to detect inflammation in the kidney tissue; one study showed that this test had an area under receiver operating characteristic curve (AUC) of 0.75 for AIN diagnosis although less than a third of the patients in this study underwent a kidney biopsy to confirm the diagnosis [8].

\section{Results from Yale AIN Study}

We compared various clinical, laboratory, urine dipstick, and microscopy features between biopsy-proven, adjudicated AIN and ATI (Table 1). Most clinical features were comparable between AIN and ATI, except for cirrhosis which was only present in patients with ATI. Laboratory features were also comparable between AIN and ATI, including the degree of renal dysfunction, blood eosinophil levels, and urine albumin. However, patients with AIN tended to have lower hemoglobin levels. Urine dipstick features were also comparable between the 2 groups including dipstick leukocyte esterase levels. AIN participants tended to have more alkaline urine despite having slightly lower serum bicarbonate levels, which may indicate tubu- 
Table 1. Comparison of clinical and laboratory features between AIN and ATI

\begin{tabular}{|c|c|c|c|}
\hline Variable & $\operatorname{AIN}(n=32)$ & $\operatorname{ATI}(n=41)$ & $p$ value \\
\hline \multicolumn{4}{|l|}{ Clinical features } \\
\hline Age, years & $58(40-68)$ & $56(42-65)$ & 0.70 \\
\hline Female & $18(56)$ & $16(39)$ & 0.14 \\
\hline African-American race & $10(31)$ & $9(22)$ & 0.37 \\
\hline Diabetes & $7(22)$ & $9(22)$ & 0.99 \\
\hline Cirrhosis & $0(0)$ & $6(15)$ & 0.02 \\
\hline AKI & $18(56)$ & $26(63)$ & 0.53 \\
\hline Severe AKI (stage 2 or higher) & $2(7)$ & $8(20)$ & 0.12 \\
\hline Dialysis at biopsy & $1(3)$ & $4(10)$ & 0.26 \\
\hline \multicolumn{4}{|l|}{ Laboratory features at biopsy } \\
\hline Serum creatinine, $\mathrm{mg} / \mathrm{dL}$ & $4.4(2.8-6.3)$ & $4.1(2.4-6.7)$ & 0.71 \\
\hline Blood urea nitrogen, $\mathrm{mg} / \mathrm{dL}$ & $40(27-57)$ & $37(28-61)$ & 0.85 \\
\hline Hemoglobin level, g/dL & $9.8(8.1-10.7)$ & $10.7(9.3-12.4)$ & 0.02 \\
\hline Platelet count $\left({ }^{*} 1,000\right.$ per $\left.\mathrm{mm}^{3}\right)$ & $247(202-290)$ & $211(169-253)$ & 0.07 \\
\hline Blood eosinophil count & $234(149-466)$ & $198(95-400)$ & 0.24 \\
\hline Urine albumin to creatinine, $\mathrm{mg} / \mathrm{g}$ & $157(44-1452)$ & $109(20-682)$ & 0.12 \\
\hline Serum bicarbonate level & $19.7(17-22.5)$ & $21(18.4-23.9)$ & 0.29 \\
\hline \multicolumn{4}{|l|}{ Urine dipstick } \\
\hline Specific gravity & $1.015(1.015-1.020)$ & $1.015(1.015-1.025)$ & 0.58 \\
\hline $\mathrm{pH}$ & $6(5.5-7)$ & $6(5.5-6.5)$ & 0.02 \\
\hline Protein, $\geq 2+$ & $19(59)$ & $20(49)$ & 0.65 \\
\hline Leukocytes, $\geq 2+$ & $10(32)$ & $11(28)$ & 0.71 \\
\hline \multicolumn{4}{|l|}{ Urine microscopy } \\
\hline White blood cell, $\geq 1 / \mathrm{HPF}$ & $8(27)$ & $8(22)$ & 0.63 \\
\hline White blood cell cast, $\geq 1 / \mathrm{HPF}$ & $1(3)$ & $1(3)$ & 0.88 \\
\hline RTE cell, $\geq 1 / \mathrm{HPF}$ & $14(47)$ & $12(32)$ & 0.23 \\
\hline $\mathrm{RTE}$ cast, $\geq 1 / \mathrm{HPF}$ & $2(7)$ & $4(11)$ & 0.58 \\
\hline Granular cast, $\geq 1 / \mathrm{HPF}$ & $10(33)$ & $17(46)$ & 0.29 \\
\hline Red blood cells, $>5 / \mathrm{HPF}$ & $5(29)$ & $10(39)$ & 0.54 \\
\hline Red blood cell cast, $\geq 1 / \mathrm{HPF}$ & $0(0)$ & $3(12)$ & 0.14 \\
\hline
\end{tabular}

Fisher's exact test or Wilcoxon Rank sum test.

Median (interquartile range) or $n(\%)$ shown.

HPF, high-power field; AKI, acute kidney injury; AIN, acute interstitial nephritis; ATI, acute tubular injury.

lar dysfunction that is described with AIN. Urine microscopy is often thought to be crucial in differentiating AIN from ATI. However, review of urine sediment in each case by a trained nephrologist did not reveal any significant differences between the 2 diseases. Given these findings, it is not surprising that a review of medical charts revealed that the clinician's prebiopsy diagnosis had a low AUC for postbiopsy AIN diagnosis (0.58 [0.47-0.68]).

\section{Histology}

In the absence of a noninvasive biomarker, the diagnosis of AIN relies on performing a biopsy to obtain kidney tissue for histological diagnosis. The typical findings of AIN are predominantly in the tubulointerstitium. AIN is characterized by infiltration of lymphocytes, macrophages, and eosinophils in the renal interstitium. This is also accompanied by the presence of inflammatory cells in the renal tubules ("tubulitis"). Tubular injury and interstitial fibrosis often accompany this inflammatory infiltrate. In our study, we noted that the pathologists were more likely to diagnose AIN if the biopsies had higher severity of interstitial lymphocytic infiltrate, tubulitis, and eosinophils (Table 2). Of the 79 cases with AIN on official biopsy interpretation, all 3 pathologists agreed on the diagnosis in $32(41 \%)$ cases and 2 out of 3 agreed in 23 (29\%) cases. In 24 (30\%) cases of AIN on official biopsy report, a majority of pathologists reclassified the diagnosis as not AIN. The pa- 
Table 2. Histological features associated with AIN

\begin{tabular}{lcc}
\hline Interstitial feature & Severity & Adjusted OR (95\% CI) \\
\hline Interstitial infiltrate, $\%$ & $<10$ & 1 (ref.) \\
& $11-25$ & $4.1(1.0-17.2)$ \\
Eosinophils & $>25 \%$ & $17.6(3.4-90.4)$ \\
& $0 / \mathrm{HPF}$ & 1 (ref.) \\
Tubulitis & $1-5 / \mathrm{HPF}$ & $8.1(2.1-30.8)$ \\
& $>5 / \mathrm{HPF}$ & $71.3(4.3-1,172.2)$ \\
Tubular injury, \% & None & 1 (ref.) \\
& $1-5 /$ tubule & $9.7(1.8-53.8)$ \\
& $<10$ & $1(\mathrm{ref})$ \\
& $11-25$ & $0.4(0.0-3.5)$ \\
& $>25 \%$ & $0.1(0.0-1.3)$ \\
\hline
\end{tabular}

Logistic regression model for outcome of AIN and predictors as various interstitial histological features reported by the adjudicating pathologists controlling for the pathologist and clustered at participant level.

HPF, high-power field; AIN, acute interstitial nephritis.

thologists were more likely to classify a biopsy as AIN when it was listed as the first numerical diagnosis on the official biopsy report (55\%) than when it was listed as second or third $(27 \% ; p=0.01)$. We noted a modest inter-rater agreement and kappa statistic among the pathologists for AIN diagnosis (agreement $63-70 \%$, Fleiss kappa $=0.35$ ). Such modest degree of agreement is not unique to AIN; poor agreement between raters has also been noted in other kidney pathologies and represents a challenge for clinician's interpreting a biopsy report [9].

\section{Novel Biomarkers}

Kidney biopsies for histological diagnoses pose risks for patients with AKI and may not be feasible in some individuals with increased bleeding risk [10]. The resulting delay in diagnosis leads to increasing fibrosis, and 40$60 \%$ of cases of AIN progress to CKD [1, 11]. As a result, novel, noninvasive biomarkers are needed to diagnosis AIN. Past studies showed that CD4+T-cells play an important role in the pathogenesis of AIN [12-14]. Based on the preliminary data, we selected 12 cytokines in the Th1 (interferon- $\gamma$, IL-2, IL-12), Th2 (IL-4, IL-5, IL-13), and Th9 (IL-9) pathways, as well as other generally inflammatory cytokines (TNF- $\alpha$, IL-1 $\beta$, IL-6, IL-8, IL-10) and compared the urine and plasma levels of these cytokines between AIN and ATI. We found that urine TNF- $\alpha$ and interleukin-9 were higher in AIN patients than in ATI controls (Fig. 1a), whereas the other urine or plasma cy- tokines were not consistently associated with AIN. These 2 urine biomarkers had an AUC of 0.83 (0.73-0.92) for AIN diagnosis. We also tested 2 cutoffs of urine IL-9: the first corresponding to the median value in the cohort $(0.41 \mathrm{ng} / \mathrm{g})$ and the second corresponding to the top 15\% values $(2.53 \mathrm{ng} / \mathrm{g})$. In Figure 1a and b, we show how urine IL-9 testing can help avoid a kidney biopsy at various prebiopsy probabilities of AIN. For example, if the prebiopsy probability of AIN is 0.25 , a value below 0.41 can rule out the diagnosis of AIN (posttest probability 0.07), whereas a value above 2.53 can rule in the diagnosis (posttest probability to 0.84 ). A value between these 2 cutoffs would likely require a kidney biopsy for diagnosis.

\section{Conclusions}

Differentiating between ATI and AIN, 2 common etiologies of AKI, is challenging for clinicians due to the lack of a reliable, noninvasive, diagnostic test. Establishing the diagnosis of AIN requires performing a kidney biopsy to obtain tissue for histological diagnosis, which carries risks and may not always be feasible. Novel biomarkers such as urine TNF- $\alpha$ and interleukin- 9 may be able to differentiate AIN from ATI. However, before wider clinical application, further studies are required to validate our findings in external cohorts, in AKI patients who aren't being considered for a biopsy, and in patients who do not have AKI but are nevertheless at high risk for AIN (e.g., users of PPI and immunotherapy agents). Moreover, a kidney biopsy may still be needed when biomarker results 

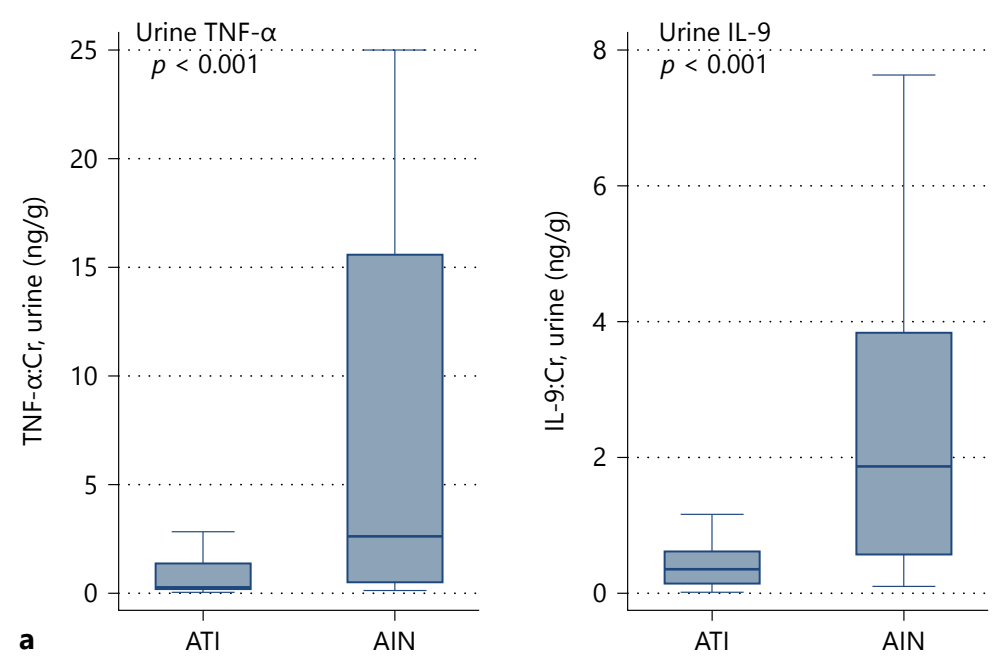

Fig. 1. Tumor necrosis factor- $\alpha$ and interleukin-9 are biomarkers to differentiate AIN from ATI. a shows median (horizontal line), 25th and 75th percentile (box), and 5th and 95th percentile (whiskers) compared between AIN and ATI. Wilcoxon Ranksum test $p$

are equivocal or to obtain prognostic information such as degree of fibrosis which may guide therapy. Finally, these biomarkers need to be linked to patient outcomes and response to therapy.

\section{Acknowledgments}

The authors would like to thank the participants of the Yale biopsy study, without whom this study would not have been possible.

Diagnosing AIN values are shown in red. b, c show posttest probability of AIN at various pretest probabilities at 2 cutoffs of IL-9. AIN, acute interstitial nephritis; ATI, acute tubular injury; IL, interleukin.

\section{Disclosure Statement}

C.R.P. and D.G.M. are named inventors in a provisional patent number 62/716,465 titled "System and methods for diagnosing AIN."

\section{Funding Sources}

This work was supported by the National Institutes of Health (K23DK117065 to D.G.M., P30DK079310 to D.G.M. and C.R.P.; UG3-DK114866 to CRP). The content is the responsibility of the 
authors alone and does not necessarily reflect the views or policies of the Department of Health and Human Services, nor does mention of trade names, commercial products, or organizations imply endorsement by the U.S. Government.

\section{Author Contributions}

C.R.P. and D.G.M. fulfill the ICMJE Criteria for Authorship and take full responsibility for the review.

\section{References}

1 Raghavan R, Eknoyan G. Acute interstitial nephritis - a reappraisal and update. Clin Nephrol. 2014 Sep;82(3):149-62.

2 Moledina DG, Wilson FP, Pober JS, Perazella MA, Singh N, Luciano RL, et al. Urine TNF- $\alpha$ and IL-9 for clinical diagnosis of acute interstitial nephritis. JCI Insight. 2019 May;4(10): 4.

3 Muriithi AK, Leung N, Valeri AM, Cornell LD, Sethi S, Fidler ME, et al. Clinical characteristics, causes and outcomes of acute interstitial nephritis in the elderly. Kidney Int. 2015 Feb;87(2):458-64.

4 Bhaumik SK, Kher V, Arora P, Rai PK, Singhal M, Gupta A, et al. Evaluation of clinical and histological prognostic markers in druginduced acute interstitial nephritis. Ren Fail. 1996 Jan;18(1):97-104.

5 Chu R, Li C, Wang S, Zou W, Liu G, Yang L. Assessment of KDIGO definitions in patients with histopathologic evidence of acute renal disease. Clin J Am Soc Nephrol. 2014 Jul;9(7): 1175-82.
6 Fogazzi GB, Ferrari B, Garigali G, Simonini P, Consonni D. Urinary sediment findings in acute interstitial nephritis. Am J Kidney Dis. 2012 Aug;60(2):330-2.

7 Wu Y, Yang L, Su T, Wang C, Liu G, Li XM. Pathological significance of a panel of urinary biomarkers in patients with drug-induced tubulointerstitial nephritis. Clin J Am Soc Nephrol. 2010 Nov;5(11):1954-9.

8 Graham F, Lord M, Froment D, Cardinal H, Bollée G. The use of gallium-67 scintigraphy in the diagnosis of acute interstitial nephritis. Clin Kidney J. 2016 Feb;9(1):76-81.

9 Liapis H, Gaut JP, Klein C, Bagnasco S, Kraus E, Farris AB 3rd, et al.; Banff Working Group. Banff Histopathological Consensus Criteria for Preimplantation Kidney Biopsies. Am J Transplant. 2017 Jan;17(1):140-50.

10 Moledina DG, Luciano RL, Kukova L, et al. Kidney Biopsy-Related Complications in Hospitalized Patients with Acute Kidney Disease. Clin J Am Soc Nephrol. 2018 Nov 7;13(11): 1633-40.
11 Muriithi AK, Leung N, Valeri AM, Cornell LD, Sethi S, Fidler ME, et al. Biopsy-proven acute interstitial nephritis, 1993-2011: a case series. Am J Kidney Dis. 2014 Oct;64(4): 558-66.

12 Spanou Z, Keller M, Britschgi M, Yawalkar N, Fehr T, Neuweiler J, et al. Involvement of drug-specific $\mathrm{T}$ cells in acute drug-induced interstitial nephritis. J Am Soc Nephrol. 2006 Oct;17(10):2919-27.

13 Zand L, Monaghan M, Griffin BR, Wagner SJ, Criaci IM, Kamal A, et al. The role of type I hypersensitivity reaction and IgE-mediated mast cell activation in acute interstitial nephritis. Clin Nephrol. 2015 Sep;84(3):13844.

14 D'Agati VD, Theise ND, Pirani CL, Knowles DM, Appel GB. Interstitial nephritis related to nonsteroidal anti-inflammatory agents and beta-lactam antibiotics: a comparative study of the interstitial infiltrates using monoclonal antibodies. Mod Pathol. $1989 \mathrm{Jul}$; 2(4):390-6. 